\title{
Overweight and Obesity among the Adolescent School Students in Belgaum City
}

Tika Ram Gurung, ${ }^{1}$ Neginhal Gurung VL²

${ }^{1}$ SANN Institute of Nursing, Shipal, Chabhil, ${ }^{2}$ Nobel College, Sinamangal, Kathmandu, Nepal.

\section{ABSTRACT}

Introduction: The World Health Organization defines obesity as a 'global epidemic'. An overweight adolescent has a 70\% chance of becoming obese. Overweight and obese adolescents are at higher risk for developing long-term chronic diseases. So the present study objective is to estimate the prevalence of overweight and obesity among adolescent school students

Methods: A sample size of 300 adolescent students was taken to do a cross-sectional study. Body weight and height were recorded and body mass index was categorized based on age-and sexspecific cut-off values as per International Obesity Task Force reference growth charts. Proportions of obese, overweight and underweight children were calculated and subjected to chi-square and logistic regression tests at the $\mathrm{p}<0.05$ significance level.

Results: The overall prevalence of overweight and obesity in the present study were $12 \%$ and $3.3 \%$ respectively. The highest prevalence was among those aged 15 years i.e. $36.7 \%$. The prevalence of overweight and obesity was higher among the subject who did not practice physical activity and whose family income was more than 10,000 per month.

Conclusions: Prevalence of overweight and obesity among adolescents student in Belgaum city was high which is statistically associated with less physically activity and monthly income of parent.

Keywords: body mass index; life style; overweight and obesity.

\section{INTRODUCTION}

Overweight and obesity are an important public health issue worldwide. The World Health Organization (WHO) defines obesity as a 'global epidemic'. ${ }^{1}$ WHO projection for 2015 estimates that over 1.5 billion people will be overweight, of which children are expected to constitute about $10 \%$. An overweight adolescent has a $70 \%$ chance of becoming obese. ${ }^{1}$ Overweight adolescents are at higher risk for developing longterm chronic conditions including adult onset diabetes mellitus, coronary heart disease, orthopaedic disorders and respiratory disease. ${ }^{1}$

In India, non-communicable diseases (NCDs) accounted for $40 \%$ of all hospital stays and $35 \%$ of all outpatient visits in $2004 .^{3}$ Also, chronic diseases are estimated to account for $53 \%$ of all deaths and $44 \%$ of disability-

Correspondence: Dr. Tika Ram Gurung, SANN Institute of Nursing, Shipal, Chabhil, Kathmandu, Nepal. Email: gtikagurung261@ gmail.com, Phone: +91-7760623368. 
adjusted life-years (DALYs) lost in 2005. ${ }^{2}$ Once considered a problem only in high-income countries, overweight and obesity are now dramatically rising in low- and middle-income countries like India, Nepal, Srilanka, Bangladesh etc., particularly in urban settings. More than $75 \%$ of overweight and obese children live in low- and middle-income countries. ${ }^{3}$

So this study was done with the objective to estimate the prevalence of overweight and obesity among adolescent school students and to assess the relation between overweight, obesity and life style at Belgaum, Karnataka, India

\section{METHODS}

A cross-sectional study was conducted among the adolescent school students of St.Paul's High school (Boys School) at Belgaum District between ages 13-16 years in the March 2010. The school was selected by using purposive sampling procedure keeping in view the operational feasibility. The total numbers of high school students were 540. The classes were categorized into three strata based on class i.e. 8th, 9th and and 10th. The proportional sample size was determined and 300 participants were selected using stratified random sampling technique. The sample size of 300 was calculated using $4 \mathrm{pq} / \mathrm{d} 2$ based on the prevalence of overweight as $25 \%$ as reported by an earlier study in Delhi, ${ }^{4}$ absolute error $5 \%$ and $q(100-p)=75 \%$.

The ethical clearance of study protocol was taken from the institutional Ethics Committee of KLE University, Belgaum. Before conducting the study, permission was obtained from school's principal and ascent was obtained from each participant. Data was collected by using semi-structured, pre-tested questionnaire from each participant.

Body weight was recorded to the nearest $0.1 \mathrm{~kg}$ using a standardized calibrated scale with subjects barefoot and wearing light clothing. Height was measured to the nearest $0.1 \mathrm{~cm}$ with standardized wall-mounted height boards with a sliding head piece according to the protocol: no shoes; heel together; child's heels, buttocks, shoulders and head touching the vertical wall surface with line of sight aligned horizontally and nothing over the head. We included the adolescent studying in class 8-10 class aged 13 to 1 years. We excluded adolescent having chronic illness and who could not stand.

All the variables were coded and entered into SPSS 16 th version; appropriate statistical analyses were performed using the same software. BMI was categorized based on age and sex-specific cut-off values of the International obesity task force (IOTF) reference growth charts. Prevalence of obesity, overweight and underweight children were calculated and subjected to chi-square and logistic regression tests at the $p<0.05$ significance level.

\section{RESULTS}

\section{General characteristics of the study population:}

In the study sample of 300, $110(36.7 \%)$ adolescent belonged to 15 years of age and $52(17.3 \%)$ were 13 years of age. $200(66.7 \%)$ adolescent were Hindus followed by Christian and Muslim 54 (18\%) and 46 (15.3\%) respectively. $65 \%$ study subject belonged to nuclear family and for 237 (79.5\%) and 203 (67.7\%) father and mother of the subject got higher education respectively. The main source of family income was business in $125(41.7 \%)$ and $9(3 \%)$ had agriculture. In the present study, the prevalence of overweight was $12 \%$, prevalence of obesity was $3.3 \%$ (Table 1 ).

\begin{tabular}{|c|c|}
\hline Classification of BMI & Number (\%) \\
\hline Underweight & $86(28.7)$ \\
\hline Normal & $168(56)$ \\
\hline Overweight & $36(12)$ \\
\hline Obese & $10(3.3)$ \\
\hline Total & $300(100)$ \\
\hline
\end{tabular}

Relationship of BMI with different factors

\begin{tabular}{|llllll|}
\hline \multicolumn{2}{|l}{ Table 2. Body Mass Index (BMI) and Physical Activity. } \\
\hline Physical Activity & Underweight & Normal & Overweight & Obesity & Total \\
NO & $3(13.0 \%)$ & $10(43.5 \%)$ & $8(34.8 \%)$ & $2(8.7 \%)$ & $23(7.7 \%)$ \\
YES & $83(30.0 \%)$ & $158(57.0 \%)$ & $28(10.1 \%)$ & $8(2.9 \%)$ & $277(92.3 \%)$ \\
Total & $86(28.67 \%)$ & $168(56 \%)$ & $36(12 \%)$ & $10(3.33 \%)$ & $300(100 \%)$ \\
\hline
\end{tabular}

$2=15.687, \mathrm{DF}=2, \mathrm{P}=0.0004$ 


\begin{tabular}{|llllll|}
\hline \multicolumn{7}{|l}{ Table 3. Body Mass Index (BMI) and Family Type. } \\
\hline Family Type & Underweight & Normal & Overweight & Obesity & Total \\
Joint & $40(38.1 \%)$ & $48(45.7 \%)$ & $13(12.4 \%)$ & $4(3.8 \%)$ & $105(35 \%)$ \\
Nuclear & $46(23.6 \%)$ & $120(61.5 \%)$ & $23(11.8 \%)$ & $6(3.1 \%)$ & $195(65 \%)$ \\
Total & $86(28.7 \%)$ & $168(56 \%)$ & $36(12 \%)$ & $10(3.3 \%)$ & $300(100 \%)$ \\
\hline
\end{tabular}

$2=8.138, D F=2, P=0.017$

The prevalence of overweight and obesity was significantly higher in those adolescent who did not practice physical activity that was $34.8 \%$ and $8.7 \%$ respectively as compared to those who practiced physical activity that was $10.1 \%$ and $2.9 \%$ respectively (Table 2 ). The prevalence of overweight and obesity were higher in joint family than nuclear that is $12.4 \%$ and $3.8 \%$ respectively and it was statistically significant (Table 3 ). Overweight and obesity was the higher $(12.4 \%$ and $3.8 \%)$ among the adolescents who belonged to joint family as compared to nuclear family and which was statistically significant $(p=0.017)$ with Chi-square test but not significant with logistic regression. Overweight and obesity were found higher among the vegetarian adolescents $(12.7$ and 5.1$)$ as compared to mixed dietary adolescent (11.8 and 2.7) but statistically not significant $(p=0.660)$. Likewise the overweight (16.5) and obesity (4.5) were higher among 15 years old adolescent but statistically not significant $(p=0.217)$.

\begin{tabular}{|l|l|l|l|l|l|}
\hline \multicolumn{2}{|l|}{ Table 4. Body Mass Index (BMI) and Monthly Family Incomes. } \\
\hline Income/Month & Underweight & Normal & Overweight & Obesity & Total \\
\hline$>10,000$ & $48(25.3 \%)$ & $105(55.3 \%)$ & $32(16.8 \%)$ & $5(2.6 \%)$ & $190(63.3 \%)$ \\
$<10,000$ & $38(34.5 \%)$ & $63(57.3 \%)$ & $4(3.6 \%)$ & $5(4.5 \%)$ & $110(36.7 \%)$ \\
Total & $86(28.7 \%)$ & $168(56 \%)$ & $36(12 \%)$ & $10(3.3 \%)$ & $300(100 \%)$ \\
\hline
\end{tabular}

$2=7.937, \mathrm{DF}=2, \mathrm{P}=0.019$

In this study the prevalence of overweight was inversely associated with family income (Table 4). The prevalence of overweight and obesity was inversely associated with family history but that was statistically not significant $(p=0.744)$.

Overweight and obesity is $5.15(\mathrm{Cl}=2.1-12.65)$ time higher among physically inactive adolescents than physically active adolescent student as well 2.71 $(\mathrm{Cl}=1.25-5.86)$ time higher among adolescent student whose parent monthly income is more than 10,000 as compare to less than 10,000 and 4.94 times higher among adolescent who watched TV but statistically not significant with $p$ value of 0.05 using univariate logistic regression. The result of univariate logistic regression was supported by multivariate logistic regression after

adjusting.

\begin{tabular}{|llll|}
\hline Table 5. Univariate Logistic Regression. & & & \\
\hline Risk Factors & Un adjusted OR & Class Interval & P Value \\
Physical Activity: NO/YES & 5.15 & $2.1-12.65$ & .000 \\
Watching TV: Yes/NO & 4.91 & $0.65-37.18$ & 0.123 \\
Socio-economic status: $>10000 /<10000$ & 2.71 & $1.25-5.86$ & 0.011 \\
\hline
\end{tabular}

\begin{tabular}{|llll|}
\hline Table 6. Multivariate Logistic Regression. & & & \\
\hline Risk Factors & Adjusted OR & Class Interval & P Value \\
Physical Activity: NO/YES & 4.51 & $1.82-11.21$ & 0.001 \\
Socio-economic status: $>10000 /<10000$ & 2.43 & $1.11-5.531$ & 0.026 \\
\hline
\end{tabular}




\section{DISCUSSION}

Obesity is becoming an increasingly important public health problem affecting all age groups; both sexes and developed as well as developing countries. The present studies of 13-17-year-olds adolescent boys have a high prevalence of overweight and obesity. Of the total, $12 \%$ were overweight and $3.3 \%$ were obese. Other studies support our findings related to overweight/obesity among adolescents. Ramachandran et al, 2002 reported 4.5 per cent in low income schools and 22 per cent in better-off schools of Chennai. ${ }^{5}$ Kapoor et al, 2003 reported prevalence of obesity and overweight to be 7.8 and 13.4 per cent from Delhi. ${ }^{6}$ Studies from rural areas mainly emphasize on under nutrition and data on overweight/obesity are not available. However, Deshmukh et al, 2006 reported prevalence of overweight/ obesity to be 2.2 per cent in rural area of Wardha district using the same definition. ${ }^{7}$ The environmental factors contributing to this are rapid urbanization and changes in lifestyles. This study analyzed the association between BMI and physical activity in adolescent and revealed that as physical activity decreases, the BMI increases. In the present study, the important determinants of the overweight/ obesity were less physical activity and high monthly family income. Joint family was significantly associated with overweight and obesity using chi-square test but not with Logistic regression. All these three factors except physical activity were family characteristics. This implies the importance of the family characteristics in the causation or predisposition of an individual to overweight/obesity. All these factors are related with affluence and sedentary lifestyle. Due to unsafe roads, lack of free space for playing and increased television viewing, computer use has made life sedentary. Giammattei, Blix, Marshak, Wollitzer, \& Pettitt, (2003) also reported that children who spent more time watching television had a higher BMI and a higher per cent of body fat and physically inactive. In the present study the watching TV is marginally associated. ${ }^{8}$ Thus there is a need to raise awareness not only among children but also parents to reduce the epidemic of high $\mathrm{BMI}$ (overweight/obesity), because this is associated with increased cholesterol and blood sugar levels and dyslipidaemias which ultimately lead to different chronic non communicable diseases. An earlier study on nutrition and physical exercise education in schools was able to show improvement in indices of fitness and body fat levels, ${ }^{9}$ supporting the proposal that physical activity must be stressed in the school environment. ${ }^{10}$ This will help in inculcating healthy among adolescent at an early age. The limitation of present study was the study done only in only one school of Belgaum city among boy students.

\section{CONCLUSIONS}

Prevalence of overweight and obesity among adolescents student in Belgaum was $12 \%$ and $3.3 \%$ which was statistically associated with lack of physically activity and high parent monthly income. There is an urgent need to implement strategies for prevention and control of overweight and obesity among the adolescents school student.

\section{ACKNOWLEDGEMENTS}

We would like to thank Mr. Rev. Fr. Geraid Furtado, Principal and Anthony John Dsouza, all the teachers and students of St. Pauls High School, Belgaum. We would like to thank all the faculty and staff members of Department of public health for their most valuable support and guidance in implementation of the study.

\section{REFERENCES}

1. Sharma A, Sharma K, Mathur KP. Growth pattern and prevalence of obesity in affluent school children of Delhi. Public Health Nutr. 2007;10(5):485-91.

2. Upadhyay RP. An overview of the burden of non communicable disease in India. Iran J Public Health. 2012;41(3):1-8.

3. World Health Organization. Obesity and overweight [Online]. Geneva: World Health Organization; 2007 [Cited 2009 June 30]. Available from URL:http:// www.who.int/mediacentre/factsheets/fs311/en/.
4. Kapil U, Singh $P$, Pathak $P$, Dwivedi SN, Bhasin S. Prevalence of obesity among affluent adolescent school children in Delhi. Indian Pediatr. 2002;39(5):449-52.

5. Ramachandran A, Snehalatha C, Vinitha R, Thayyil M, Kumar CK, Sheeba L, et al. Prevalence of overweight in urban Indian adolescent school children. Diabetes Res Clin Pract. 2002;57(3):185-90.

6. Sethi M, Kapoor P. Obesity. New Delhi: Voluntary Health Association of India; 2003. 
7. Deshmukh PR, Gupta SS, Bharambe MS, Dongre AR, Maliye C, Kaur S, et al. Nutritional status of adolescents in rural Wardha. Indian $\mathrm{J}$ Pediatr. $2006 ; 73(2): 15-7$.

8. Giammattei J, Blix G, Marshak $\mathrm{HH}$, Wollitzer AO, Pettitt DJ. Television watching and soft drink consumption: associations with obesity in 11-to 13-year-old school children. Arch Pediatr Adolesc Med. 2003;157(9):882-6.
9. Epstein LH. Effects in treatment of childhood obesity. Int J Obes. 1995;19:117-21.

10. Shetty $P$. Childhood obesity in developing countries. NFI Bulletin. 1999;20:1-4. 